\title{
Spatiotemporal distribution and population at risk of soil-transmitted helminth infections following an eight-year school- based deworming programme in Burundi, 2007-2014
}

Mohamad Assoum ${ }^{1,2,3^{*}}$, Giuseppina Ortu ${ }^{5}$, Maria-Gloria Basáñez ${ }^{6}$, Colleen Lau ${ }^{1,7}$, Archie C. A. Clements ${ }^{7}$, Kate Halton ${ }^{8}$, Alan Fenwick ${ }^{4}$ and Ricardo J. Soares Magalhães ${ }^{1,2}$

\begin{abstract}
Background: Investigating the effect of successive annual deworming rounds on the spatiotemporal distribution of infection prevalence and numbers at risk for soil-transmitted helminths (STHs) can help identify communities nearing elimination and those needing further interventions. In this study, we aim to quantify the impact of an 8-year mass drug administration (MDA) programme (from 2007 to 2014) on the spatiotemporal distribution of prevalence of STH infections and to estimate the number of school-aged children infected with STHs in Burundi.

Methods: During annual longitudinal school-based surveys in Burundi between 2007 and 2011, STH infection and anthropometric data for a total of 40,656 children were collected; these data were supplemented with data from a national survey conducted in 2014. Bayesian model based geostatistics (MBG) were used to generate predictive prevalence maps for each STH species and year. The numbers of children at-risk of infection per district between 2008 and 2014 were estimated as the product of the predictive prevalence maps and population density maps.

Results: Overall, the degree of spatial clustering of STH infections decreased between 2008 and 2011; in 2014 the geographical clusters of all STH infections reappeared. The reduction in prevalence was small for Ascaris lumbricoides and Trichuris trichiura in the centre and central north of the country. Our predictive prevalence maps for hookworm indicate a reduction in prevalence along the periphery of the country. The predicted number of children infected with any STH species decreased substantially between 2007 and 2011, but in 2014 there was an increase in the predicted number of children infected with A. lumbricoides and T. trichiura. In 2014, the districts with the highest predicted number of children infected with A. lumbricoides, T. trichiura and hookworms were Kibuye district $(n=128,903)$, Mabayi district $(n=35,302)$ and Kiremba $(n=87,511)$, respectively.

Conclusions: While the MDA programme in Burundi resulted in a reduction in STH prevalence, this reduction was spatiotemporally heterogeneous, with some pockets of high prevalence remaining, suggesting that treatment coverage and complementary interventions should be evaluated to improve impact.
\end{abstract}

Keywords: Spatiotemporal modelling, Soil-transmitted helminths, Predictive risk mapping, Number of infections

\footnotetext{
* Correspondence: m.assoum@uq.edu.au

'Children's Health and Environment Program, Child Health Research Centre,

The University of Queensland, Brisbane, Australia

${ }^{2}$ UQ Spatial Epidemiology Laboratory, School of Veterinary Science, The

University of Queensland (Gatton Campus), Via Warrego Highway QLD,

Gatton 4343, Australia

Full list of author information is available at the end of the article
}

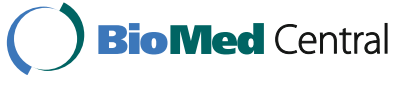

(c) The Author(s). 2017 Open Access This article is distributed under the terms of the Creative Commons Attribution 4.0 International License (http://creativecommons.org/licenses/by/4.0/), which permits unrestricted use, distribution, and reproduction in any medium, provided you give appropriate credit to the original author(s) and the source, provide a link to the Creative Commons license, and indicate if changes were made. The Creative Commons Public Domain Dedication waiver (http://creativecommons.org/publicdomain/zero/1.0/) applies to the data made available in this article, unless otherwise stated. 


\section{Background}

Soil-transmitted helminth (STH) infections are intestinal nematode infections that affect approximately 1.6 billion people around the world, with the majority of infections occurring in resource-poor settings [1]. Since the signing of the London Declaration on Neglected Tropical Diseases (NTDs) in 2012, programmes for the control of STH infections and other NTDs have received renewed support from the pharmaceutical industry, the scientific community and key development agencies and stakeholders [2]. Reductions in prevalence of infection and associated morbidity can be achieved by successive mass drug administration (MDA). More recently, it has been argued that to further control and reach elimination targets, MDA campaigns would need to be integrated with water, sanitation and hygiene (WASH) programmes [3]. While MDA is seen as a cost-effective intervention to achieve morbidity control, rapid re-infection means that it can be ineffective at reducing transmission, especially for populations living perennially in STH-contaminated environments. Morbidity control through long-standing MDA programmes can be undermined by geographical disparities in drug coverage and drug efficacy and by socioeconomic conditions that limit the access and adequate utilisation of water and sanitation infrastructure [4].

The Schistosomiasis Control Initiative (SCI) has been actively involved with the planning, implementation and continued monitoring and evaluation of anthelmintic MDA programmes using albendazole (ALB) and mebendazole (MEB) in 16 sub-Saharan African (SSA) nations including Burundi. From 2007 until 2014, SCI supported an MDA programme in Burundi [5], primarily targeting school-aged children (SAC) and pregnant women. In 2007, a pilot longitudinal study was launched in 12 schools, followed in 2008 by an extension study, in which an additional 19 schools across the country were added [6]. The longitudinal study aimed to assess the impact of MDA on STH control in Burundi, and found that the overall prevalence of STH infection was statistically significantly reduced over the programme's duration. However, this investigation also found that programmatic disruption (due to political and civil unrest in late 2009 through to 2010) resulted in substantially reduced levels of treatment coverage for that period, with a consequent detectable resurgence in STH prevalence. This highlighted the pressing need for STH control interventions to not rely solely on MDA, a strategy that may not be sustainable in the long term and which crucially requires achieving high levels of treatment coverage and adherence. This notion has been supported by numerous other studies [7-9], which indicate that in some endemic areas with high transmission, high intensity of infection may persist, requiring integration of MDA with WASH if elimination is to be achieved.
Predictive prevalence mapping based on spatial models that include environmental drivers of infection has been widely used to identify areas in SSA where communities are at highest risk of STH infection and thus deworming campaigns should be targeted [10-13]. Most studies have focused on estimating the spatial variation of indirect morbidity indicators, such as prevalence and intensity of infection [11, 14]. In the case of Burundi, predictive prevalence maps were produced in 2007 to focus treatment delivery based on areas of high uncertainty of high infection prevalence [15]. The study found that predictive prevalence mapping was indeed an effective tool for guiding MDA implementation to maximise deworming efficiency [5]. However, the impact of successive (annual) MDA rounds on the spatiotemporal variation of prevalence of STH infections such as the ensuing 8-year MDA programme in Burundi [6] has not been investigated. In our previous study, we found that disruption in the delivery of MDA, for example as a result of social unrest, may have contributed to the observed rebound in STH infection prevalence [6]. Furthermore, we found that the most common co-infections noted were $A$. lumbricoides and T. trichiura which peaked in 2008 at $2.72 \%$. However rates of co-infections dropped substantially over the course of the MDA, with coinfections making up less than $2 \%$ per year following 2008. However, the impact of the MDA programme on the spatiotemporal distribution of prevalence of STH infection is largely unknown, and this understanding may have implications for the achievement of the overall intervention goal. Thus mapping heterogeneity in prevalence of infection over time is important, as it allows us to identify areas where MDA has been systematically successful and, more importantly, areas where it may have failed and where further MDA campaigns may be needed.

In the present study, we aim to: (i) quantify the impact of an 8-year MDA programme (from 2007 to 2014) on the geographical distribution of STH infection prevalence, and (ii) estimate the spatiotemporal variation in the number of STH-infected children following the 8year programme. Our ultimate goal is to identify areas in Burundi where the impact of MDA has been systematically suboptimal at reducing prevalence and number of infections; this will help support the planning of further studies within these areas to understand the determinants of programme coverage and efficacy. Furthermore, it will also support the planning of further programmatic activities.

\section{Methods}

Data collection on STH infection

The protocol for data collection for the 2007-2011 surveys has been reported elsewhere [5]. In brief, the 20072011 surveys were conducted in conjunction with the 
delivery of the MDA programme. Data collected included child's age, sex, height, weight, and parasite egg count by STH species. Stool samples were taken from 100 children (approximately 50 boys and 50 girls) per school [6]; each year, samples were collected in May and the MDA round was delivered in June. The diagnostic approach using the Kato-Katz method was detailed in our previous paper [6]. During the 2014 survey, similar data collection protocols comparable to those of the 2008-2011 period were used [6]. In 2014, all 12 schools from the pilot study plus 14 out of the 19 schools from the extension study were re-assessed to evaluate the prevalence and intensity of STH infection after 7 years of annual MDA [6]. In each school in 2014, 50 pupils aged between 12 and 16 years were recruited, with the exception of one pilot study school in which 100 pupils were recruited $[5,6]$. In the 2008-2011 cohort, students were aged between 5 and 18 yrs. In 2014, the Ministry of Health, with the support of the Schistosomiasis Consortium for Operational Research and Evaluation (SCORE), conducted a national survey. Further details on the 2014 national survey have been reported elsewhere [6].

A single stool sample was collected from each child and duplicate slides were prepared [6]. Diagnosis of STH infection was performed using the Kato-Katz technique by trained local ground staff [16-18]. If a single egg of a given parasite species was found, the child was considered positive for that parasite species. Egg counts were used to detail the intensity of infection.

Geographical coordinates of each school were recorded using hand-held global positioning system (GPS) units. Overall prevalence of infection was calculated for each school and for each parasite species. These summary data were plotted in a geographical information system (GIS) (ArcMap version 10.3, ESRI, Redlands, CA, USA).

Infection data were gathered and collected from the same 31 schools during 3 years (2008, 2009 and 2011); however, due to civil unrest, only 12 out of the 31 were surveyed in 2010. In 2014, 26 out of the 31 schools were surveyed due to staffing issues. A total of 40,656 children were sampled over the 8 years. For the 2014 survey, height, weight and blood haemoglobin levels were not measured.

\section{Environmental and population data}

Environmental influences on STH species, such as $A$. lumbricoides and T. trichiura, are well known. Land surface temperature (LST), soil type, and distance to a water bodies influence the survival of parasite eggs in the environment, and therefore determine the intensity of exposure [19]. Equally, the transmission of hookworm species is determined by climate and landscape, as their larvae burrow into the soil to survive in more favourable micro-environments [20]. Electronic data for a normalised difference vegetation index (NDVI) for a $30 \times 30 \mathrm{~m}$ grid cell resolution were obtained from LandSAT 5 and 8 satellite images via the Google Earth Engine (GEE) database (Additional file 1: Table S1). Elevation data with a $30 \times 30 \mathrm{~m}$ grid resolution, generated by a digital elevation model (DEM) from the Advanced Space-borne Thermal Emission and Reflection Radiometer (ASTER) Global Digital Elevation Model (GDEM), were obtained. LST data were also obtained from the ASTER system with a $500 \times 500 \mathrm{~m}$ resolution. Precipitation data were collected from WorldClim with $1 \times 1 \mathrm{~km}$ grid resolution. Remotely-sensed data for LST and NDVI were recorded monthly from 2007 to 2014 and a new annual raster file was created. The locations of large perennial inland water bodies were obtained from the Food and Agriculture Organization of the United Nations [21], and the distance to perennial inland water bodies (DPWB) was estimated for each survey location in the GIS. A $5 \times 5 \mathrm{~km}$ resolution population density surface derived from the Global Rural-Urban Mapping Project (GRUMP) beta product was obtained from the Centre for International Earth Science Information Network (CIESIN) of the Earth Institute at Columbia University [22]. Values at each survey location for all environmental datasets were extracted in the GIS.

\section{Statistical analyses \\ Non-spatial models of STH infection}

We assessed the temporal variation in environmental variables between 2007 and 2011, and it was found that the environmental variables did not vary significantly between years. As such, only the 2011 values were used for analyses (Additional file 1: Table S2). The relationship between the prevalence of infection with each parasite for each of the 31 schools and the arithmetic mean of each environmental variable at the school location was evaluated using scatter plots and lines of best fit. If the relationship was found to be linear, then the variable was included in the univariable and multivariable analysis as a fixed effect. Non-linear relationships were explored using linear regression; however, we did not consider any transformation for our final models. To identity the best set of uncorrelated predictor environmental covariates, the Pearson's correlation coefficient was calculated for all pairs of environmental variables at all data locations for all years.

Fixed-effects binomial logistic regression models of prevalence of infection for each STH parasite species were developed in Stata version 10.1 (Stata Corporation, College Station, TX, USA). All univariable models included the individual-level variables age and sex as fixed effects and environmental covariates including either NDVI, LST, precipitation, DPWB or elevation. In the 
univariable analysis, Wald's $P$-value of 0.2 was used to select variables to be included in the final multivariable models for each parasite species. Multivariable analysis was conducted including age and sex as fixed effects in the models and all selected environmental variables as fixed effects. Using a backward stepwise process of variable selection, variables with a $P$-value greater than 0.05 were excluded from the final multivariable model. However, if the coefficient of a given variable changed by more than one quarter of the value of the model preceding, due to the removal of the variable, then the removed variable was deemed to be a confounder and was retained in the final model. If a confounder was identified, the model with the lowest Akaike information criterion (AIC) was selected.

\section{Analysis of residual spatial dependence}

Residuals from the final multivariable models for each STH species were extracted for each survey year and residual spatial dependence was estimated using semivariograms, constructed using the geoR package of the statistical software $\mathrm{R}$ (The R Foundation for Statistical Computing) [23]. Semivariograms are defined by three parameters, namely the nugget, the range and the sill. The sill is constituted by the sum of the partial sill and the nugget. The partial sill and nugget correspond, respectively, to the components of residual variation that are spatially structured and unstructured variation (e.g. random error). The range indicates the average size of clusters of STH prevalence. The proportion of the variance in the data that is due to geographical location can be estimated by dividing the partial sill by the sill. A spatial trend in prevalence of infection is present when the sill of a semivariograms is not attained within a reasonable range, indicating the range is very large relative to the study area. Propensity for clustering is calculated by the partial sill divided by the sum of the partial sill and the nugget.

\section{Spatial risk prediction and model validation}

A total of 40,656 individual observations of STH infection status across all years were included in the analysis.

Spatial modelling was conducted on data collected between 2007 and 2011 and separately for 2014. Spatial prediction of STH prevalence was performed for each year using model-based geostatistics [24] with the Bayesian statistical software, OpenBUGS version 1.4 (Medical Research Council Biostatistics Unit, Cambridge, UK and Imperial College London, London, UK). All models included time, individual and environmental covariates as fixed effects plus a geostatistical random effect, in which spatial autocorrelation between locations was modelled using an exponentially decaying autocorrelation function. To improve identifiability and model convergence, all environmental variables were standardised by subtracting the mean and dividing by the standard deviation. The resulting regression coefficients for these variables represent the effect of a change of one standard deviation in these variables.

The outputs of Bayesian models, including parameter estimates and spatial prediction at unsampled locations, are distributions termed "posterior distributions". The posterior distributions represent fully the uncertainties associated with the parameter estimates. We summarised the posterior distributions in terms of the posterior mean and standard deviation. Predicted prevalence estimates were categorised into 6 categories for visualisation: category 1 indicates very low STH prevalence $(<2 \%)$; category 2 indicates low prevalence $(2-10 \%)$; category 3 indicates moderate STH prevalence (10-20\%); category 4 indicates moderately-high prevalence $(20-50 \%)$; category 5 indicates high prevalence (50-80\%; and category 6 very high prevalence $(>80 \%)$. Prediction uncertainty was defined by the standard deviation and was categorised into 3 categories: low uncertainty (standard deviation $<0.2$ ), moderate uncertainty (standard deviation 0.2-0.5) and high uncertainty (standard deviation $>0.5$ ). Estimation of surface areas was conducted in ArcGIS using raster calculators and zonal statistics.

The predictive accuracy of the prevalence of infection models was assessed using the mean prediction error, the mean absolute error and the correlation coefficient between the predicted and observed values. The mean error quantifies the bias of the predictor, and the mean absolute error provides a measure for the association between the observed and predicted values. The correlation between the observed and predicted data was tested using Pearson's correlation coefficient (Additional file 1: Table S3).

\section{Estimation of number of school age children at risk of STH infection}

Population density maps were multiplied by the predicted prevalence maps in ArcGIS version 10.3 (ESRI, Redlands, CA) to estimate the number of SAC predicted to be infected with each of the STH species per year per district. Population data for Burundi were obtained from CIESIN2000, and population growth rates for years 2005 to 2014 were obtained from the World Bank [25]. To estimate population for each survey year, the base population figure from 2011 was multiplied by the population growth rate.

\section{Results}

Dataset for analysis

All variables, with the exception of precipitation (for which a quadratic relationship was explored), had a linear relationship with STH infection prevalence. 
Precipitation was subsequently excluded from the final multivariable model because it was not statistically significantly associated with prevalence of infection. Initial univariate analyses demonstrated that LST and elevation were highly correlated, with a Pearson's correlation coefficient of 0.9 . However, the $P$-value and AIC scores for LST was lower than the $P$-value for elevation and for that reason elevation was excluded from the multivariable analysis. In the multivariate models, only LST and NDVI were found to be associated $(P>0.05)$ with the prevalence of all parasites at each survey location.

\section{Residual spatial variation}

The residual semivariograms for $A$. lumbricoides prevalence of infection indicate that, after accounting for the effect of environmental covariates, infections were clustered during the years 2010 (average cluster size: $68 \mathrm{~km}$; propensity for clustering: 80\%) and 2011 (average cluster size: $77 \mathrm{~km}$; propensity for clustering: 93\%) (Additional file 1: Figure S1a-e). For T. trichiura, residual geographical clustering was present in 2008 (average cluster size: $52 \mathrm{~km}$; propensity for clustering: 100\%) and 2009 (average cluster size: $61 \mathrm{~km}$; propensity for clustering: 100\%) (Additional file 1: Figure S2a-e, Table S4). For hookworm infections, clustering was only found in 2008 and spatial trends in 2009 and 2010 (average cluster size: $22 \mathrm{~km}$; propensity for clustering: $75 \%$ ) (Additional file 1: Figure S3a-e, Table S4). In 2014, residual semivariograms for $A$. lumbricoides and hookworm prevalence demonstrated trends in spatial dependence, whilst no spatial dependence was evident for $T$. trichiura.

\section{Spatial risk prediction}

Model effect sizes for each parasite between 2008 and 2011 and 2014 can be found in Additional file 1: Table S5. Predictive prevalence maps for both A. lumbricoides (Fig. 1) and T. trichiura (Fig. 2) demonstrate that the western region, the eastern border, the south-eastern border region and the north-eastern region of the country experienced a gradual reduction in STH prevalence from 2008 until 2014. Our predictive prevalence maps for A. lumbricoides show that between 2008 and 2014, the central south-western and north-western regions of the country areas demonstrated continued moderately high prevalence (>20\% and less than $50 \%$ ) after several rounds of MDA were observed. Furthermore, areas to the north-west of the country experienced an increase in prevalence in 2014. Our predictive prevalence maps for T. trichiura show that in the central-northern region of the country there was a slight reduction in prevalence. This region, however, also maintained higher prevalence values (>10\% and less than $20 \%$ ) than the surrounding regions; this is particularly evident between 2008 and 2011. In 2014, a small region where moderate prevalence (>10\% and less than 20\%) of infection is predicted appeared in the south-western region of the country with a prevalence higher than in 2008. Our predictive

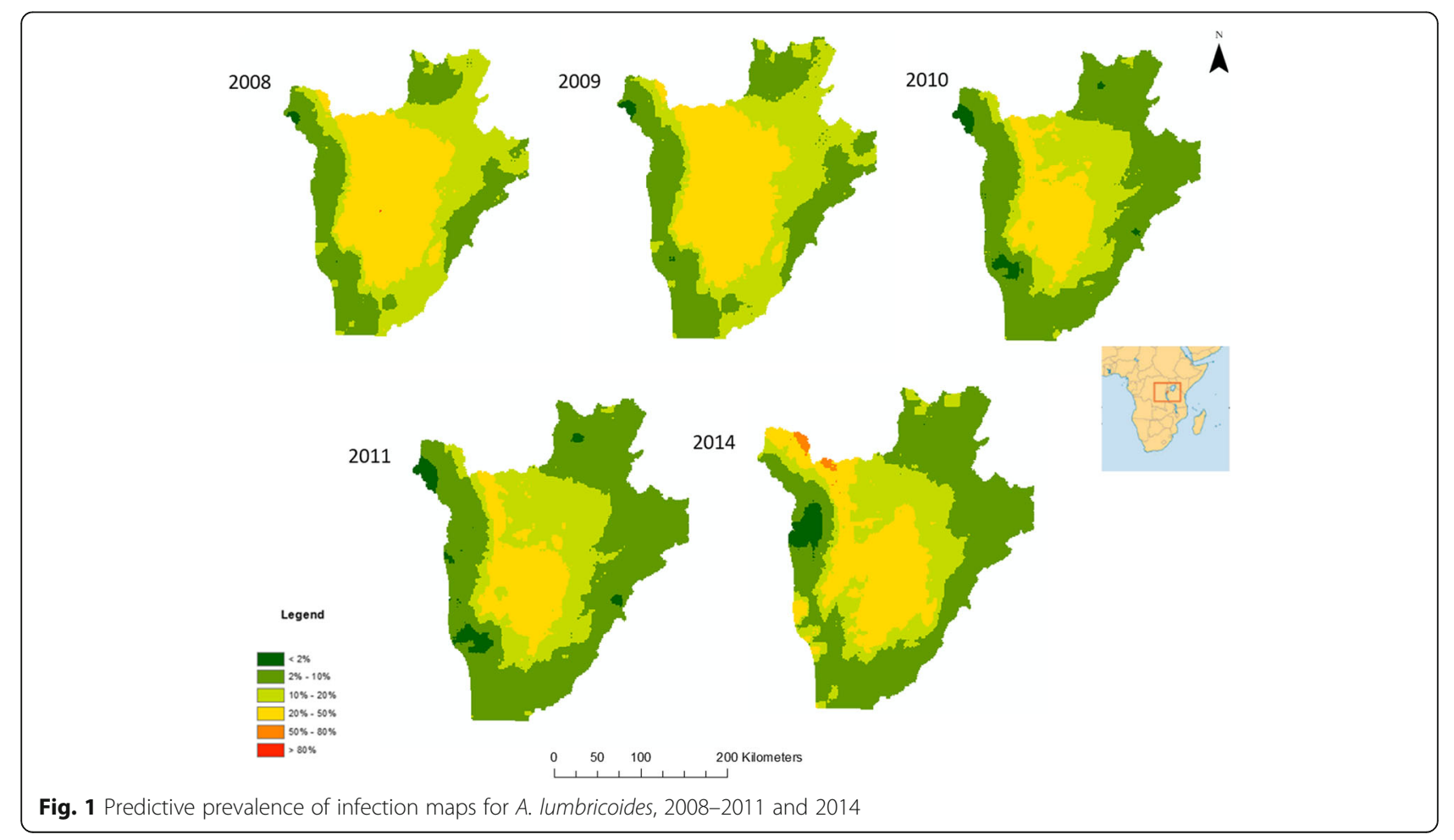



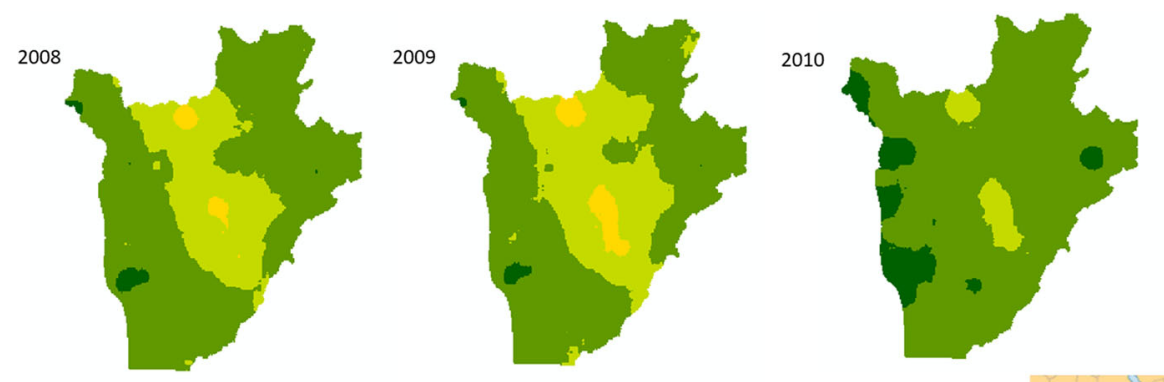

$\hat{\Lambda}$
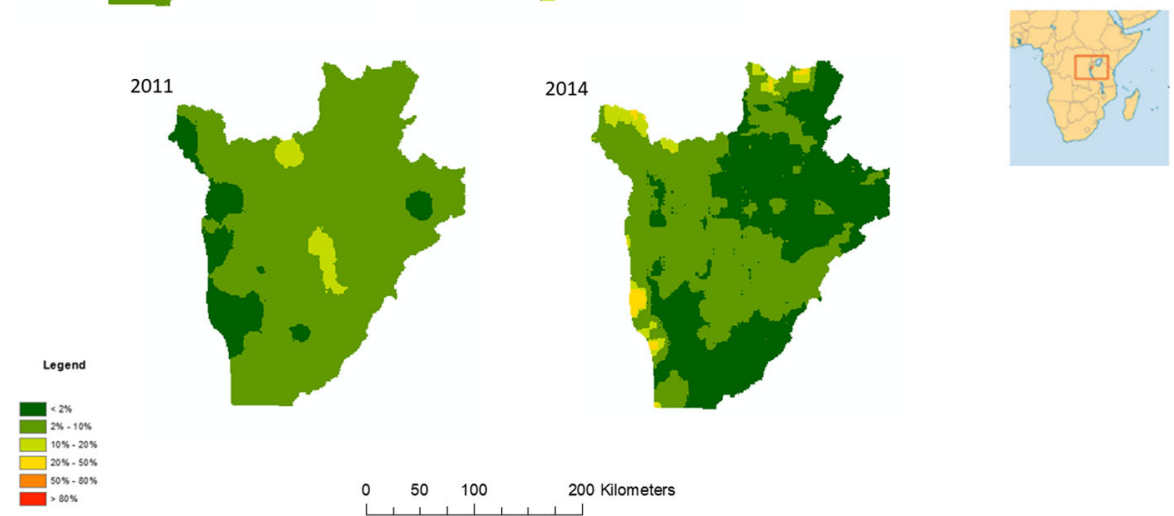

$0 \quad 50 \quad 100$

200 Kilometers

Fig. 2 Predictive prevalence of infection maps for T. trichiura, 2008-2011 and 2014

prevalence maps for hookworm (Fig. 3) indicate that in 2008 the west and eastern regions had the highest predicted prevalence of infection (between 20 and 50\%); by 2011 these regions observed a significant reduction in prevalence (predicted prevalence reaching 10-20\%).
However, in 2014 prevalence of hookworm infection was predicted to be as high as $50 \%$ in the north southwest and small pockets in the east of the country.

For all parasite species, there was a substantial reduction in the total surface area of high and moderate
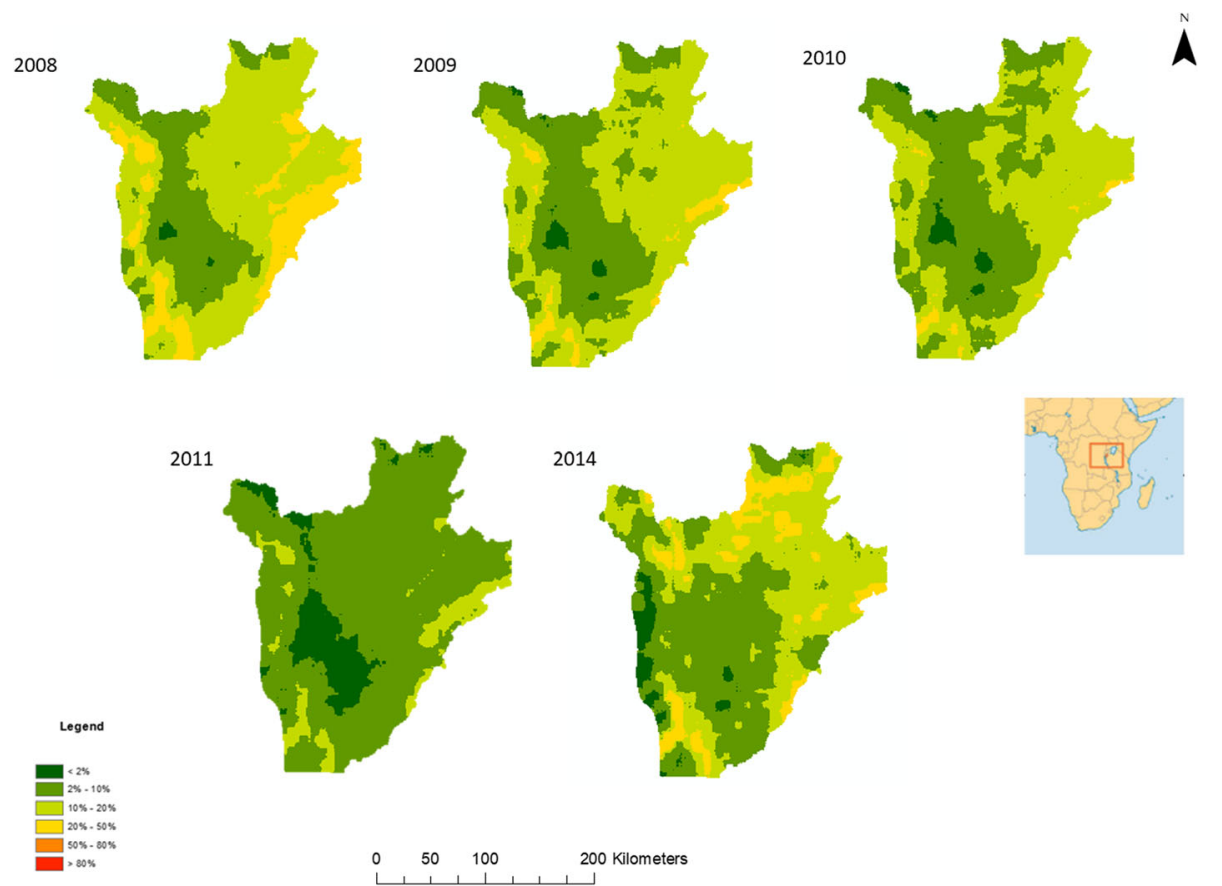

Fig. 3 Predictive prevalence of infection maps for hookworm, 2008-2011 and 2014 
prevalence categories between 2008 and 2011 with a resurgence in 2014 (Table 1). For all parasites our results indicate a decrease in the overall surface area of moderate and high prevalence categories from $15,734 \mathrm{~m}^{2}$ in 2008 to $4277 \mathrm{~m}^{2}$ in 2011. The results for A. lumbricoides demonstrate a reduction in the surface areas for high infection prevalence (>50\% and less than $80 \%$ ) and moderate infection prevalence ( $>20 \%$ and less than $50 \%$ ) between 2008 and 2011 and an increase in 2014, with an overall total surface area of $10,310 \mathrm{~km}^{2}$ in 2008, $4277 \mathrm{~km}^{2}$ in 2011, and $6802 \mathrm{~km}^{2}$ in 2014. These changes were accompanied by a substantial increase in surface area of regions within the low infection prevalence category. For T. trichiura, there was a reduction in surface area for high (>50\% and less than $80 \%)$ and moderately high infection prevalence ( $>20 \%$ and less than 50\%) from $416 \mathrm{~km}^{2}$ in 2008 to $0 \mathrm{~km}^{2}$ in 2011 and an increase of $286 \mathrm{~km}^{2}$ in 2014. For T. trichiura there were no very high prevalence categories (> 80\%) from 2008 to 2014, with all high prevalence areas (>50\% and less than $80 \%$ ) transitioned to a moderately high prevalence $(>20 \%$ and less than $50 \%$ ) status. Nearly all moderate prevalence categories (>10\% and less than 20\%) transitioned into low prevalence $(>2 \%$ and less than $10 \%$ ) categories. In 2008 , very few areas were classified with very low

Table 1 Changes in surface area (in $\mathrm{km}^{2}$ ) of prevalence of infection categories in Burundi for 2008-2011 and 2014

\begin{tabular}{|c|c|c|c|c|c|}
\hline & 2008 & 2009 & 2010 & 2011 & 2014 \\
\hline \multicolumn{6}{|c|}{ Prevalence of A. lumbricoides infections } \\
\hline$<2 \%$ & 78 & 130 & 586 & 791 & 605 \\
\hline $2-10 \%$ & 8348 & 9510 & 15,017 & 15,404 & 13,214 \\
\hline $10-20 \%$ & 9104 & 8799 & 7113 & 7370 & 7046 \\
\hline $20-50 \%$ & 10,311 & 9404 & 5127 & 4277 & 6802 \\
\hline $50-80 \%$ & 2 & 0 & 0 & 0 & 176 \\
\hline$>80 \%$ & 0 & 0 & 0 & 0 & 0 \\
\hline \multicolumn{6}{|c|}{ Prevalence of $T$. trichiura infections } \\
\hline$<2 \%$ & 346 & 231 & 2634 & 3189 & 13,006 \\
\hline $2-10 \%$ & 19,844 & 17,679 & 23,901 & 23,804 & 13,730 \\
\hline $10-20 \%$ & 7237 & 8915 & 1307 & 849 & 821 \\
\hline $20-50 \%$ & 416 & 1018 & 0 & 0 & 286 \\
\hline $50-80 \%$ & 0 & 0 & 0 & 0 & 0 \\
\hline$>80 \%$ & 0 & 0 & 0 & 0 & 0 \\
\hline \multicolumn{6}{|c|}{ Prevalence of hookworm infections } \\
\hline$<2 \%$ & 165 & 491 & 701 & 3223 & 1061 \\
\hline $2-10 \%$ & 7770 & 10,890 & 12,908 & 22,344 & 12,305 \\
\hline $10-20 \%$ & 15,262 & 15,299 & 13,757 & 2276 & 11,397 \\
\hline $20-50 \%$ & 4646 & 1162 & 476 & 0 & 3079 \\
\hline $50-80 \%$ & 0 & 0 & 0 & 0 & 0 \\
\hline$>80 \%$ & 0 & 0 & 0 & 0 & 0 \\
\hline
\end{tabular}

prevalence $(<2 \%)$ (surface area $346 \mathrm{~km}^{2}$ ); however, by 2014 most areas in the country were classified with very low prevalence (surface area 13,006 $\mathrm{km}^{2}$ ). For hookworm there was a substantial decline in moderate prevalence surface area between 2008 and 2011, from $4646 \mathrm{~km}^{2}$ to $0 \mathrm{~km}^{2}$; however, a resurgence of the moderate and high prevalence categories was evident in 2014, with a total surface area of $3079 \mathrm{~km}^{2}$.

For A. lumbricoides, regions in the north, south and east of the country showed low to very low prediction uncertainty. Low (standard deviation below 0.2) to moderate (standard deviation between 0.2-0.5) uncertainty was evident in the central and western regions of the country (Additional file 1: Figure S4). Predictions for $T$. trichiura had low to very low uncertainty throughout the country. Patches of low to moderate uncertainty were evident in the centre of the country between 2008 and 2011, whilst in 2014 moderate uncertainty corresponded closely to areas of moderate prevalence of infection (Additional file 1: Figure S5). For hookworm, uncertainty was low across the country between 2008 and 2011. However, in 2014, moderate uncertainty was evident in the northern, eastern and southern regions of the country (Additional file 1: Figure S6).

\section{Model validation}

The models for A. lumbricoides prevalence demonstrated low mean absolute error (MAE) for all years (ranging between 0.03 and 0.06 ) with high Pearson's correlation coefficients (PCC) (ranging between 0.84 and 0.98 ) for all years (Additional file 1: Table S3). The models for $T$. trichiura prevalence demonstrated low mean absolute error for all years (MAE between 0.01 and 0.04) with high Pearson's correlation coefficients (ranging between 0.93 and 0.94) for 2008, 2009 and 2011. Correlation was weak in 2010 and 2014, ranging between 0.16 and 0.47 (Additional file 1: Table S3). The models for hookworm prevalence also demonstrated low mean absolute error (ranging between 0.03 and 0.04) and high Pearson's correlation coefficients (ranging between 0.74 and 0.83 ) for all years (Additional file 1: Table S3).

\section{Spatial heterogeneity in the number of school age children infected with STH}

An overall reduction in the number of infected SAC was evident for all parasite species from 2008 to 2011 in all districts. In 2014, an estimated total of 4,098,816 children were infected with at least one species, either $A$. lumbricoides (Fig. 4), T. trichiura (Fig. 5) or hookworms (Fig. 6). A reduction in the predicted number of children infected with $A$. lumbricoides was evident from 2008 to 2011, with the highest predicted number of infected children in 2008 being 119,619 infected children in the Gitega district for A. lumbricoides and in 2014 in the Kibuye 

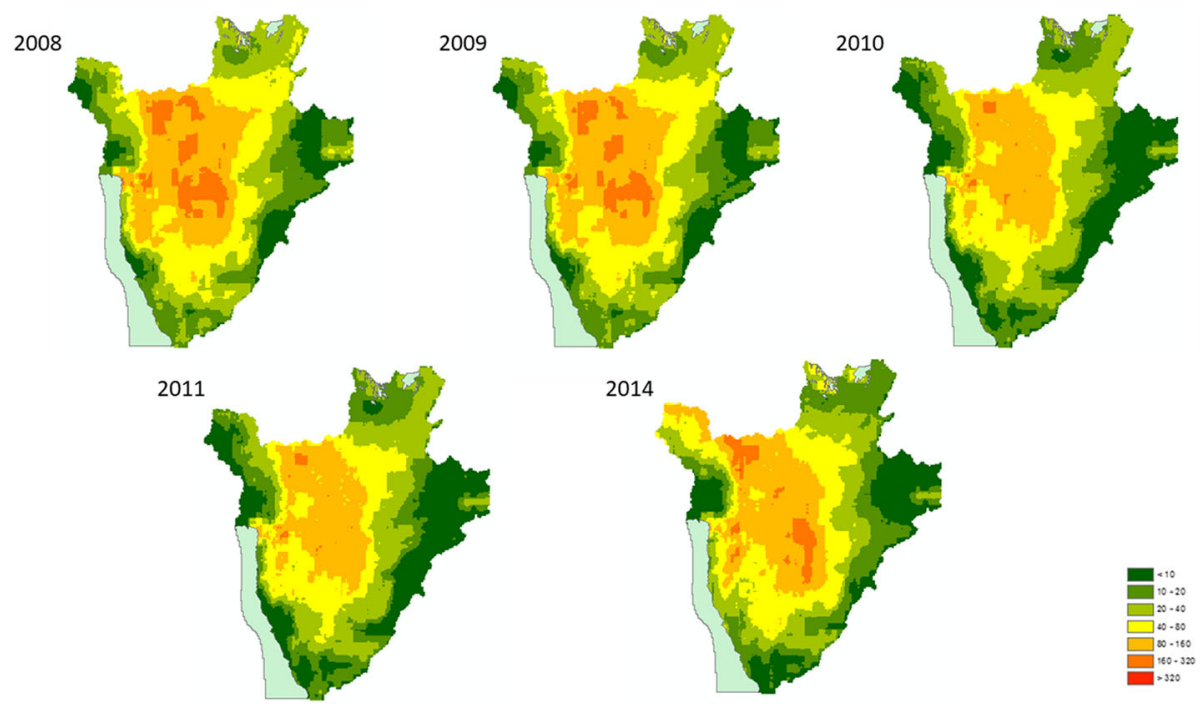

Fig. 4 Predicted number of children aged 5 to 15 infected with Ascaris lumbricoides in 2008-2011 and 2014

district, with 128,903 children infected. For T. trichiura, and in 2008, the district with the highest number of infected children was Ngozi, with 65,669 infected children. In 2014, the Mabayi district was predicted to have the highest number of SAC with $T$. trichiura, with 35,302 infected children. In 2008, hookworm infection was highest in the Muyinga district, with an estimated 66,828 children infected with $N$. americanus/A. duodenale. In 2014, this figure increased to 87,511 in Kiremba. Overall, the number of children infected with hookworm saw a $4.9 \%$ increase between 2008 and 2014 (Additional file 1: Table S6).

\section{Discussion}

In our previous study we reported marked variation in STH prevalence between the different years of the $\mathrm{Bu}$ rundian MDA programme, thus justifying a more formal assessment of the spatiotemporal distribution of STH prevalence in Burundi [6]. Here we quantify, for the first time, the impact of an 8-year MDA programme on the spatiotemporal variation in infection prevalence and predict the number of children infected with each STH species over the course of the programme. The maps and infection burden estimates presented here can help intervention planning to best
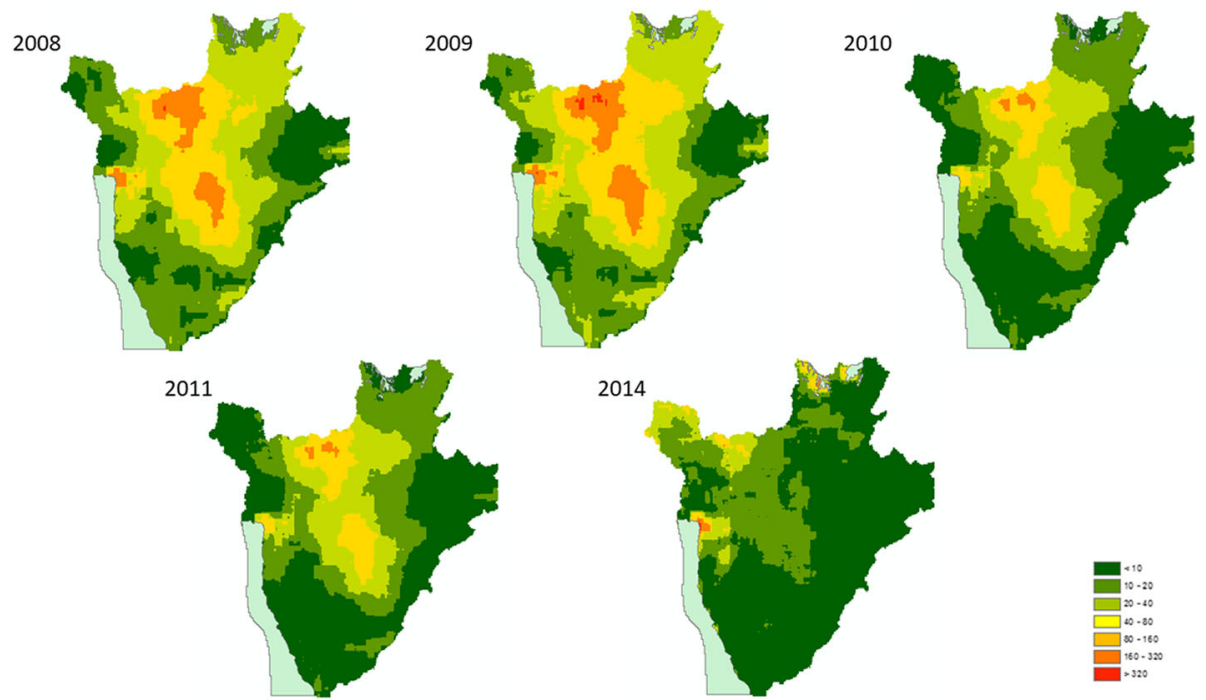

Fig. 5 Predicted number of children aged 5 to 15 infected with Trichuris trichiura in 2008-2011 and 2014 

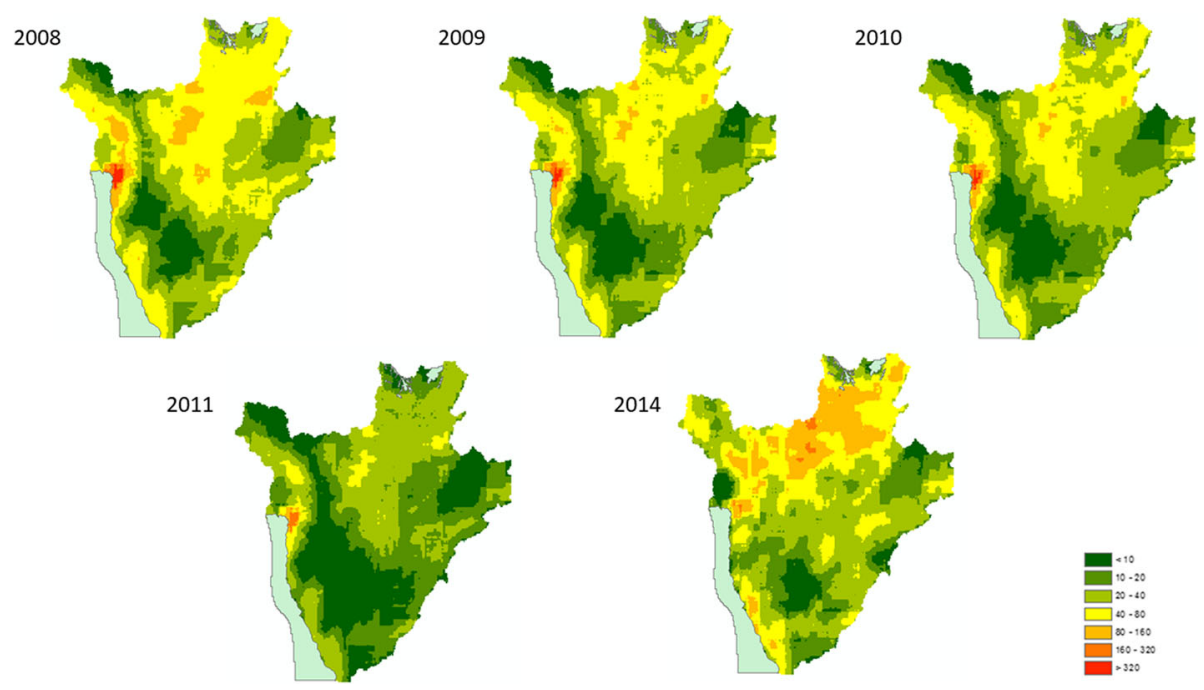

Fig. 6 Predicted number of children aged 5 to 15 infected with hookworm in 2008-2011 and 2014

utilise resources to ensure that areas that are most at risk of STH infection are targeted [10, 26-28]. Our maps could also be useful to guide the control programme in Burundi on how to best reach transmission control and elimination goals by linking with transmission dynamics models [29].

The effect of socio-economic, climate and physical environment on STH infections is well known $[19,30]$ and has been used to investigate the spatial distribution of STH infections [20, 31, 32]. In our multivariable models of prevalence of A. lumbricoides and T. trichiura infections, we found that areas with higher prevalence of infection were associated with temperatures between 25 and $37{ }^{\circ} \mathrm{C}$ and lower vegetation indexes. This is in line with existing evidence indicating that $A$. lumbricoides and T. trichiura species require temperatures below $37{ }^{\circ} \mathrm{C}$ and low NDVI values to facilitate their survival and transmission [19].

\section{Spatiotemporal variation in STH infection prevalence 2008-2014}

Quantifying the relative change in the geographical clustering in different parasite species over the duration of successive annual MDAs can help determine the population effect of anthelmintic treatment and the likelihood of particular areas achieving elimination. It is expected that, as a result of MDA, clusters of high prevalence of infection will be reduced in size as areas less resistant to treatment shift their level of endemicity to moderate and low prevalence of infection. As a result of successive MDA rounds between 2008 and 2010, prevalence of STH infection was reduced [6]; the results of the present study demonstrate that the observed reduction in prevalence between 2009 and 2011 was accompanied by a concurrent reduction in the geographical clustering of STH infections, particularly for T. trichuris and hookworm infections, as evidenced by a reduction in the propensity for clustering (from 2009 to 2014 in the case of both species). This finding is corroborated by our predictive prevalence maps which indicate a reduction in the prevalence of $T$. trichiura infection in central districts of the country and along the periphery of the country in the case of hookworm where these infections where principally distributed.

This suggests that during 2008-2011 geographical patterns of T. trichiura and hookworm infections shifted from widespread high-endemicity clusters into less defined prevalence clusters but still exhibited some residual spatial trend in infection. In the case of A. lumbricoides, despite the reduction in prevalence of infection, the spatial patterns from 2008 to 2011 remained relatively stable, with highly endemic areas present in the central districts.

However, resurgence in prevalence of infection was detected in 2014 in the northwest and southwest regions of the country for A. lumbricoides and T. trichiura and in the northwest, southwest, east and northeast for hookworm. This increase resulted in the re-emergence of the moderate infection prevalence category, with $A$. lumbricoides also experiencing resurgence in the high prevalence category. The precise reasons for this resurgence are largely unknown since the longitudinal study concluded in 2011 and follow-up surveys were not conducted until 2014.

Areas of civil unrest were mainly documented in the western, south-western, north-western and north-eastern regions of the country [33, 34]. The original 12 pilot school sites (situated mainly in the west of the country [6]) remained relatively safe, while in all extension survey 
sites (more evenly distributed throughout the country [6]), treatment was halted in 2010 due to disruption by the civil unrest. Disruption of the MDA programme in affected areas is likely to have impacted on the spatial distribution of infections due to uneven coverage of MDA. Central and eastern areas received the majority of the internallydisplaced population and there was a notable division between treated and untreated populations there. The 12-month treatment disruption and the observed impacts highlight the potential impact of population dynamics and contextualising population movement in the context of STH transmission; the importance of developing spatially-structured dynamic models in addition to spatially-structured geostatistical models; and the need to develop WASH infrastructure that would change prevailing transmission conditions more sustainably.

The above regions' higher initial prevalence rates, for example in Kibumbu, Gitega and their immediate districts [6], could also be a factor in resurgence. Even currently hypo-endemic areas may have an increased risk of resurgence or reintroduction if they were formerly hyper-endemic, thus emphasizing that MDA programmes alone are not sustainable in maintaining low morbidity in the long term in areas prone to destabilisation. Moreover, the overall geographical distribution of hookworm species appeared to be inversely associated with that of A. lumbricoides and T. trichiura. This, together with the fact that the different STH species are characterised by different age profiles of infection, highlights the need to understand the macro- and micro-epidemiology of the STH component infections separately.

\section{Spatial variation in treatment needs following 8-year MDA in Burundi}

Combining infection prevalence maps with estimates of population numbers has allowed us to: (i) estimate the temporal variation in the predicted number of infected SAC over different years of the MDA programme in Burundi; (ii) identify areas where reductions in these numbers were more or less pronounced and, therefore, to highlight areas where the number of infected SAC remained roughly unaltered; and (iii) predict geographically the number of SAC infected for 2014, the year during which a "national reassessment" of the programme was conducted. By taking population density into account, our results demonstrate that in the case of $A$. lumbricoides and T. trichiura, the central and central northern regions of Burundi should be the focus of future MDA programmes, as these contain communities where the number of infected children is predicted to be highest. However, in the case of hookworm infection, the eastern western region as well as the northern regions should be of particular focus. Predictive infection distribution maps are an important extension that allow for effective and programmatically helpful decisionsupport tools to target treatments to populations in greatest need. An important extension to our work could involve coupling our models to dynamic disease transmission models that account for internal population migration/displacement.

\section{Limitations}

A number of limitations need to be considered when interpreting our results. First, our results indicated that areas of moderate uncertainty are co-distributed with areas of moderate to high prevalence. This may be so because our data had few cases of moderate and heavy infections from 2009 onwards. One of the principal purposes of evaluating the level of uncertainty in mapped outputs is to demonstrate areas where further investigations are needed [35]. Second, the presence and intensity of STH infections are determined by poor hygiene and sanitation, and socio-economic demographics [3, 36, 37], but data indicating the state of hygiene practices and the availability of sanitation infrastructure in the study districts were not available. Third, although we endeavoured to obtain remotely-sensed data with the highest possible resolution, in some instances, the resolution of the data was not ideal (with pixels approximating $1 \mathrm{~km}$ by $1 \mathrm{~km}$ ). This is a limiting factor as it contributes to regression dilution bias. Similarly, population maps used in our models have been adjusted using general annual growth rates and as such they are subject to accuracy issues as annual growth rates may have not been necessarily homogenous across the entire nation. Fourth, we did not account in our modelling framework for the impact of other NTD interventions, such as treatment of onchocerciasis, which not only is community wide (rather than targeted at particular age and population groups), but also includes ivermectin, an anthelmintic which, when combined with $\mathrm{ABZ}$, has a better efficacy for $T$. trichiura than ABZ or MBZ on their own [29]. This gap may act as a critical factor influencing the differences observed between 2011 and 2014, but it was difficult to obtain programmatic data for these two programmes and the extent of their overlap with the STH programme. Finally, while our validation statistics demonstrate high correlation and low mean errors for most parasite species and years, this was not the case for T. trichiura in 2010 and 2014, where Pearson's correlation coefficient was poor (i.e. $<0.7$ ). This is likely due to the fact that more than $30 \%$ of the survey locations had no T. trichiura infections for the target age and sex subpopulation of our prediction model.

\section{Conclusions}

Follow-up parasitological surveys, as well as MBG mapping updates throughout the programme, have been 
used to monitor the overall progress achieved with the STH MDA intervention in Burundi from 2007 to 2014 in terms of changes in the spatiotemporal clustering of prevalence, surface area of endemicity levels and numbers of children at risk. Together with a decrease in prevalence, a decrease in infection clustering was also observed, suggesting that successive MDA rounds were successful at reducing infection clusters [38], shifting infection patterns from clusters of high to moderate infection levels to more dispersed cases of infection. This was evident for all parasite species over the course of the MDA programme. Furthermore, the small-scale geographical distribution of STH species also changed over the course of this programme. The number of infected SAC varied geographically across the years and for the different parasite species. Finally, the success of the MDA programme appears to be very sensitive to perturbations to the programme and possibly to internal migration with and areas rebounding to higher prevalence levels in a matter of a couple of years.

\section{Additional file}

\begin{abstract}
Additional file 1: Table S1. Environmental data summary. Table S2. Correlation coefficients for NDVI and LST, 2007-2011. Table S3. Model validation results, mean prediction error, absolute prediction error and Pearson's correlation coefficient for all parasites, 2008-2011 and 2014. Table S4. Average size of clusters and propensity of clustering per parasite per year. Table S5. Model effect sizes for all parasites, 2008-2011 and 2014. Table S6. Total number of infected children per year per parasite 2008-2011 and 2014. Figure S1. Residual semivariograms for prevalence of infection with Ascaris lumbricoides in Burundi for years 2007-2011 and 2014. Figure S2. Residual semivariograms for prevalence of infection with Trichuris trichiura in Burundi for years 2007-2011 and 2014. Figure S3. Residual semivariograms for prevalence of infection with hookworm in Burundi for years 2007-2011 and 2014. Figure S4. Posterior mean standard deviation of predicted prevalence of infection with Ascaris lumbricoides in Burundi for 2008-2011 and 2014. Figure S5. Posterior mean standard deviation of predicted prevalence of infection with Trichuris trichiura in Burundi for 2008-2011 and 2014. Figure S6. Posterior mean standard deviation of predicted prevalence of infection with hookworm in Burundi for 2008-2011 and 2014. (DOCX 761 kb)
\end{abstract}

\begin{abstract}
Abbreviations
95\% Cl: 95\% confidence interval; ABZ: Albendazole; AIC: Akaike information criterion; ASTER: Advanced spaceborne thermal emission and reflection radiometer; AUC: Area under curve; CIESIN: Center for International Earth Science Information Network; DEM: Digital elevation model; DPWB: Distance to perennial water body; GDEM: Global digital elevation map; GEE: Google Earth Engine; GIS: Geographical information systems; GLM: Generalised linear models; GPS: Global positioning system; GRUMP: Global rural urban mapping project; LST: Land surface temperature; MAE: Mean absolute error; MBG: Modelbased geostatistics; MBZ: Mebendazole; MDA: Mass drug administration; NDVI: Normalized differential vegetation index; NTD: Neglected tropical diseases; PCC: Pearson's correlation coefficient; ROC: Receiver operating characteristic; SAC: School-aged children; SCl: The Schistosomiasis Control Initiative; SCORE: Schistosomiasis Consortium for Operational Research and Evaluation; SSA: Sub-Saharan Africa; STH: Soil-transmitted helminths; WASH: Water and sanitation and hygiene; WHO: World Health Organization
\end{abstract}

\section{Acknowledgements}

We thank all the partners involved in this programme (the Ministry of Health in Burundi, Schistosomiasis Control Initiative, CBM, the Global Network
Neglected Tropical Disease Control, and Geneva Global), school directors, laboratory technicians and nurses that have made this work possible.

\section{Funding}

The reported monitoring and evaluation programme was financially supported by Geneva Global and the Global Network for Tropical Diseases (GNTD) in collaboration with the Sabine Institute, and by the Schistosomiasis Control Initiative, which provided also technical assistance. The reported national mapping survey conducted in 2014 was funded by the Schistosomiasis Consortium for Operational Research and Evaluation (SCORE) and Schistosomiasis Control Initiative.

\section{Availability of data and materials}

According to institutional agreements with the Minister of Health in Burundi, full dataset can be shared upon the Ministry of Health's permission. Therefore, the full dataset cannot be shared at this stage.

\section{Authors' contributions}

MA, RJSM and GO conceived and designed the study. GO and team executed the study and coordinated the field work. GO supervised the data collection. MA and RJSM collated the data from all studies, cleaned it and started the data analysis. MA analysed the data. RJSM supervised the data analysis. MA wrote the first draft of the manuscript. GO, RJSM, $M G B, C L, K H, A C$ and $A F$ reviewed the manuscript critically and contributed intellectual input. MA prepared the final draft. All authors read and approved the final manuscript.

\section{Authors' information}

Mohamad Assoum: A PhD candidate at the University of Queensland, Australia focusing on the spatial epidemiology of STH infections in child populations. Background in medical geography and international public health (BSc, GradDip GIS, MIPH(hon)). Working in collaboration with SCI at Imperial College London. Giuseppina Ortu: Senior Programme Manager at Schistosomiasis Control Initiative (Imperial College, London, UK) at the time of the programme in Burundi, and currently Senior NTD specialist at Malaria Consortium (London, UK) and Honorary Research Associate at Imperial College (London, UK). Colleen Lau: Infectious disease epidemiologist and clinician, and currently an NHMRC Fellow at the Department of Global Health, Research School of Population Health, The Australian National University. Kate Halton: Lecturer in Health Economics, School of Public Health \& Social Work, Queensland University of Technology. Senior Research Fellow in Infectious Disease, Australian Centre for Health Services Innovation. María-Gloria Basáñez: Professor of Neglected Tropical Diseases at Imperial College London; member of the London Centre for Neglected Tropical Disease Research and long-standing collaborator of the Schistosomiasis Control Initiative. Alan Fenwick: Director, Schistosomiasis Control Initiative in the Department of Infectious Disease Epidemiology, Imperial College London. Archie Clements: Director, Research School of Population Health. An infectious disease epidemiologist working on neglected tropical diseases and other tropical infections. Ricardo J Soares Magalhaes: Senior Lecturer in Population Health and Veterinary Biosecurity, School of Veterinary Science, Faculty of Science, The University of Queensland. Affiliate Senior Lecturer UQ Children's Health Research Centre.

\section{Ethics approval and consent to participate}

Ethical clearance for all surveys was obtained from the Ministry of Health $(\mathrm{MoH})$ in Burundi. Written and verbal consent were obtained from the $\mathrm{MoH}$, school teachers and the parents or guardians of the children recruited in the study. Ethical clearance was also obtained by the SCI from Imperial College Research Ethics Committee (ICREC_8_2_2). Data were anonymized prior to analysis by assigning each participant a unique identification number. For all surveys, children found positive for STH infection at the time of surveys received treatment immediately. In addition, treatments were administered to all children in endemic schools during the MDA rounds, which occurred between two and four weeks after surveys were conducted.

Consent for publication

Not applicable.

Competing interests

The authors declare that they have no competing interests. 


\section{Publisher's Note}

Springer Nature remains neutral with regard to jurisdictional claims in published maps and institutional affiliations.

\section{Author details}

'Children's Health and Environment Program, Child Health Research Centre, The University of Queensland, Brisbane, Australia. ${ }^{2}$ UQ Spatial Epidemiology Laboratory, School of Veterinary Science, The University of Queensland (Gatton Campus), Via Warrego Highway QLD, Gatton 4343, Australia. ${ }^{3}$ School of Medicine, The University of Queensland, Brisbane, Australia. ${ }^{4}$ Schistosomiasis Control Initiative, Imperial College London, Department of Infectious Disease Epidemiology, School of Public Health, Faculty of Medicine (St. Mary's Campus), Norfolk Place, W2 1PG, London, UK. ${ }^{5}$ Present address: Malaria Consortium Headquarters. Development House, 56-64 Leonard Street EC2A 4LT, London, UK. ${ }^{6}$ London Centre for Neglected Tropical Disease Research, Department of Infectious Disease Epidemiology, Faculty of Medicine (St. Mary's Campus), Imperial College London, School of Public Health, Norfolk Place W2 1PG, London, UK. ' Research School of Population Health, Australian National University, Canberra, Australia. ${ }^{8}$ Queensland University of Technology, Brisbane, Australia.

\section{Received: 16 June 2017 Accepted: 29 October 2017} Published online: 23 November 2017

\section{References}

1. Pullan RL, Smith JL, Jasrasaria R, Brooker SJ. Global numbers of infection and disease burden of soil-transmitted helminth infections in 2010. Parasit Vectors. 2014;7(1):37

2. Neglected Tropical Diseases, Uniting to Combat: Iondon declaration on neglected tropical diseases. 2012. http://unitingtocombatntds.org/sites/ default/files/document/london_declaration_on_ntds.pdf. Accessed 20 Mar 2016.

3. Campbell SJ, Savage GB, Gray DJ, Atkinson J-AM, Soares Magalhães RJ, Nery SV, et al. Water, sanitation, and hygiene (WASH): a critical component for sustainable soil-transmitted helminth and schistosomiasis control. PLoS Negl Trop Dis. 2014;8:e2651.

4. Truscott JE, Turner HC, Farrell SH, Anderson RM. Soil-transmitted helminths: mathematical models of transmission, the impact of mass drug administration and transmission elimination criteria. Adv Parasitol. 2016;94:133-98.

5. Ndayishimiye O, Ortu G, Soares Magalhaes RJ, Clements A, Willems J, Whitton $J$, et al. Control of neglected tropical diseases in Burundi: partnerships, achievements, challenges, and lessons learned after four years of programme implementation. PLoS Negl Trop Dis. 2014;8:e2684

6. Ortu G, Assoum M, Wittmann U, Knowles S, Clements M, Ndayishimiye O, et al. The impact of an 8-year mass drug administration programme on prevalence, intensity and co-infections of soil-transmitted helminthiases in Burundi. Parasit Vectors. 2016;9:513.

7. Freeman MC, Clasen T, Brooker SJ, Akoko DO, Rheingans R. The impact of a school-based hygiene, water quality and sanitation intervention on soiltransmitted helminth reinfection: a cluster-randomized trial. Am J Trop Med Hyg. 2013;89:875-83.

8. Mascarini-Serra L. Prevention of soil-transmitted helminth infection. J Glob Infect Dis. 2011;3:175-82

9. Minamoto K, Mascie-Taylor CGN, Karim E, Moji K, Rahman M. Short- and long-term impact of health education in improving water supply, sanitation and knowledge about intestinal helminths in rural Bangladesh. Public Health. 2012;126(5):437.

10. Pullan RL, Gething PW, Smith JL, Mwandawiro CS, Sturrock HJ, Gitonga CW, et al. Spatial modelling of soil-transmitted helminth infections in Kenya: a disease control planning tool. PLoS Negl Trop Dis. 2011;5:e958.

11. Magalhães RJS, Biritwum N-K, Gyapong JO, Brooker S, Zhang Y, Blair L, et al. Mapping helminth co-infection and co-intensity: geostatistical prediction in Ghana. PLoS Negl Trop Dis. 2011:5:e1200.

12. Clements ACA, Garba A, Sacko M, Toure S, Dembele R, Landoure A, et al. Mapping the probability of schistosomiasis and associated uncertainty. West Africa Emerg Infect Dis. 2008;14:1629.

13. Clements ACA, Lwambo NJS, Blair L, Nyandindi U, Kaatano G, Kinunghi S, et al. Bayesian spatial analysis and disease mapping: tools to enhance planning and implementation of a schistosomiasis control programme in Tanzania. Tropical Med Int Health. 2006;11:490-503.
14. RJS M, ACA C, Patil AP, Gething PW, Brooker S. The applications of modelbased geostatistics in helminth epidemiology and control, vol. 74. England: Elsevier Science \& Technology; 2011. p. 267-96.

15. Clements A, Deville M, Ndayishimiye O, Brooker S, Fenwick A. Spatial codistribution of neglected tropical diseases in the east African great lakes region: revisiting the justification for integrated control. Tropical Med Int Health. 2010;15:198-207.

16. Assefa LM, Crellen T, Kepha S, Kihara JH, Njenga SM, Pullan RL, et al. Diagnostic accuracy and cost-effectiveness of alternative methods for detection of soil-transmitted helminths in a post-treatment setting in western Kenya. PLoS Negl Trop Dis. 2014:8:e2843.

17. Knopp S, Speich B, Hattendorf J, Rinaldi L, Mohammed KA, Khamis IS, et al. Diagnostic accuracy of Kato-Katz and FLOTAC for assessing anthelmintic drug efficacy (diagnostics and anthelmintic drug efficacy). PLoS Negl Trop Dis. 2011:5:e1036.

18. Speich B, Knopp S, Mohammed KA, Khamis IS, Rinaldi L, Cringoli G, et al. Comparative cost assessment of the Kato-Katz and FLOTAC techniques for soil-transmitted helminth diagnosis in epidemiological surveys. Parasit Vectors. 2010;3:71

19. Brooker S, Clements ACA, Bundy DAP. Global epidemiology, ecology and control of soil-transmitted helminth infections, vol. 62. England: Elsevier Science \& Technology; 2006. p. 221-61.

20. Brooker S, Clements ACA, Bundy DAP. Global epidemiology, ecology and control of soil-transmitted helminth infections. Adv Parasitol. 2006:62:221-61.

21. Nations FaAOotU. http://www.fao.org/geonetwork/srv/en/main.home.

22. University ElaC. http://sedac.ciesin.columbia.edu/gpw/global.jsp.

23. Pullan RL, Sturrock HJ, Soares Magalhaes RJ, Clements AC, Brooker SJ. Spatia parasite ecology and epidemiology: a review of methods and applications. Parasitology. 2012;139:1870-87.

24. Magalhães RJS, Barnett AG, Clements ACA. Geographical analysis of the role of water supply and sanitation in the risk of helminth infections of children in West Africa. Proc Natl Acad Sci USA. 2011;108:20084-9.

25. The World Bank. http://databank.worldbank.org/data/reports.aspx?Code=NY. GDP.PCAP.CD\&id=af3ce82b\&report_name=Popular_indicators\&populartype= series\&ispopular=y. Accessed 20 Mar 2016.

26. Brooker S, Kabatereine NB, Gyapong JO, Stothard JR, Utzinger J. Rapid mapping of schistosomiasis and other neglected tropical diseases in the context of integrated control programmes in Africa. Parasitology. 2009;136:1707-18.

27. Kabatereine NB, Standley CJ, Sousa-Figueiredo JC, Fleming FM, Stothard JR, Talisuna A, et al. Integrated prevalence mapping of schistosomiasis, soiltransmitted helminthiasis and malaria in lakeside and island communities in Lake Victoria, Uganda. Parasit Vectors. 2011;4:232.

28. Standley CJ, Adriko M, Alinaitwe M, Kazibwe F, Kabatereine NB, Stothard JR. Intestinal schistosomiasis and soil-transmitted helminthiasis in Ugandan schoolchildren: a rapid mapping assessment. Geospat Health. 2009;4:39-53.

29. Basáñez M-G, McCarthy JS, French MD, Yang G-J, Walker M, Gambhir M, et al. A research agenda for helminth diseases of humans: modelling for control and elimination. PLoS Negl Trop Dis. 2012;6:e1548.

30. Chammartin F, Scholte RGC, Guimarães LH, Tanner M, Utzinger J, Vounatsou P. Soil-transmitted helminth infection in South America: a systematic review and geostatistical meta-analysis. Lancet Infect Dis. 2013;13:507.

31. Pullan RL, Bethony JM, Geiger SM, Cundill B, Correa-Oliveira R, Quinnell RJ, et al. Human helminth co-infection: analysis of spatial patterns and risk factors in a Brazilian community. PLoS Negl Trop Dis 2008;2:e352.

32. Scholte RGC, Schur N, Bavia ME, Carvalho EM, Chammartin F, Utzinger J, et al. Spatial analysis and risk mapping of soil-transmitted helminth infections in Brazil, using Bayesian geostatistical models. Geospat Health. 2013;8:97-110.

33. Hoofnagle K, Rothe D. Overlooked and overshadowed: the case of Burundi. Crit Crim. 2010:18:169-89.

34. Schneider J. Lessons from Burundi's unrest. New African. 2015;551:36-8.

35. Araujo Navas AL, Hamm NAS, Soares Magalhães RJ, Stein A. Mapping soiltransmitted helminths and schistosomiasis under uncertainty: a systematic review and critical appraisal of evidence. PLoS Negl Trop Dis. 2016;10:e0005208.

36. Asaolu SO, Ofoezie IE. The role of health education and sanitation in the control of helminth infections. Acta Trop. 2003;86:283-94.

37. Blair P, Diemert D. Update on prevention and treatment of intestinal helminth infections. Curr Infect Dis Rep. 2015;17:1.

38. Cairncross S, Blumenthal U, Kolsky P, Moraes L, Tayeh A. The public and domestic domains in the transmission of disease. Tropical Med Int Health. 1996;1:27-34. 